\title{
Proof of Nernst Heat Theorem and Its Corollaries
}

\author{
Naum Tsirelman \\ Aviation Thermotechnics Thermal Power Department, \\ Ufa State Aviation Technical University, Ufa 450000, Russia \\ bkcuazs@mail.ru
}

Received 20 September 2013, Revised 07 May 2014, Accepted 26 January 2017

\begin{abstract}
It is known that the Nernst's Heat Theorem (the Third Law of Thermodynamics) having a vast number of applications was derived experimentally. In this work its theoretical proof is given outside the framework of quantum mechanical considerations using the dependence determined by R. Clausius to calculate the infinitesimal quantity of heat.
\end{abstract}

Keywords: Temperature; entropy; heat; Nernst; heat theorem.

\section{Introduction}

The W. Nernst "heat theorem" is formulated as follows: around zero thermodynamic temperature $T$, the entropies $S$ of all bodies being in the equilibrium state experience no changes and equal to each other, i.e. the entropy $S$ of any body at $T \rightarrow 0$ is constant [1-3].

The proof of this theorem is still based on the methods of information theory and statistical mechanics [1-4], and in the present work it was obtained in the framework of classical thermodynamics.

For the proof of the W. Nernst heat theorem, it seems to be appropriate to consider the peculiarities of equilibrium heat exchange processes, when the body thermodynamic temperature $T$ approaches zero.

Let's find out what the behavior of the multiplier $d S$ is in the R. Clausius formula stated below for calculation of the infinitesimal quantity of heat $\delta Q$ given the proposition of the validity of the relation at $T \rightarrow 0$ as well:

$\delta Q=T d S$.

\section{Theoretical Nernst Heat Theorem Proof}

\subsection{Case 1}

Without distinguishing the positive infinite small difference $\varepsilon>0$ of the body temperature $T$ and the cooler ( $T$ $>\varepsilon, \varepsilon \rightarrow 0)$ and the module of negative infinite small amount of rejected heat $\delta Q<0$, for $\varepsilon=|\delta Q|^{*}$ in accordance to (1), the following is obtained:

$$
\left.d S\right|_{T \rightarrow 0}=\left.\frac{\delta Q}{T}\right|_{T \rightarrow 0}=-\frac{\varepsilon}{T} .
$$

\footnotetext{
* Here and later on, during the comparison of $\varepsilon$ and $\delta Q$ values, they are considered dimensionless due to dividing their dimension values by $1 \mathrm{~K}$ and $1 \mathrm{~J}$ correspondingly
}

Let the approximation of the point $(\varepsilon, T)$ to the origin $(0,0)$ follows the curve $T=T(\varepsilon)$, which has in it the vertical tangent (Figure 1 ). Show that in the region $\Omega_{1}$ bounded by the curve and axis $0 T$, the right part of (2) approaches zero at $(\varepsilon, T) \rightarrow(0,0)$.

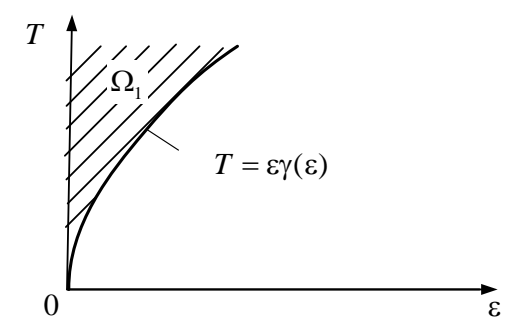

Figure 1. Monotonic dependence of temperature $T$ on $\varepsilon$.

Accounting for the condition $T>\varepsilon$, the dependence $T=T(\varepsilon)$ can be written, for example, as

$T=\varepsilon \cdot \gamma(\varepsilon)$,

where

$\gamma(\varepsilon) \in(1, \infty), \gamma(\varepsilon) \rightarrow \infty, \varepsilon \cdot \gamma(\varepsilon) \rightarrow 0$ as $\quad \varepsilon \rightarrow 0$.

The condition $\varepsilon \cdot \gamma(\varepsilon) \rightarrow 0$ can be satisfied if, particularly, $\gamma(\varepsilon)=k / \varepsilon^{1-\beta}$ at $\varepsilon<k^{1 /(1-\beta)}(k>0, \beta \in(0 ; 1)$ are constants).

In this case, the dependence of $T$ on $\varepsilon$ at the right boundary $\Omega_{1}$ is set by the functions which have in the neighborhood of the point $(0,0)$ the power form of $T=k \varepsilon^{\beta}$, for which under $\beta=1 / 2$ or $\beta=1 / 3$, the arc of the quadratic 
or cubic parabola correspondingly perform as part of the right boundary of the wedge-shaped region $\Omega_{1}$ hatched in Figure 1 , adjoining the point $(0,0)$.

It is this region around the point $(0,0)$ at $\varepsilon<k^{1 /(1-\beta)}$, the right side of Eq.(2) approaches zero at $(\varepsilon, T) \rightarrow(0,0)$.

Make sure, first of all, that if the function $T(0)=0$ then has in the point $(0,0)$ the vertical tangent:

$T^{\prime}(0)=\lim _{\varepsilon \rightarrow 0} \frac{T(\varepsilon)-T(0)}{\varepsilon-0}=\lim _{\varepsilon \rightarrow 0} \frac{T(\varepsilon)}{\varepsilon}=\lim _{\varepsilon \rightarrow 0} \frac{\varepsilon \gamma(\varepsilon)}{\varepsilon}=$

$=\lim _{\varepsilon \rightarrow 0} \gamma(\varepsilon)=\lim _{\varepsilon \rightarrow 0} k \varepsilon^{\beta-1}=+\infty$.

Next, calculate at the neighborhood of zero thermodynamic temperature the value of $d S$ at $T>\varepsilon$ and $\varepsilon \rightarrow 0$ :

$\left.d S\right|_{T \rightarrow 0}=-\frac{\varepsilon}{T}=-\frac{\varepsilon}{\varepsilon \gamma(\varepsilon)}=-\frac{1}{\gamma(\varepsilon)}=-\frac{1}{k \varepsilon^{\beta-1}}=-\frac{\varepsilon^{1-\beta}}{k}$

$=-\frac{\varepsilon^{\delta}}{k} \rightarrow 0$,

where $\delta=1-\beta \in(0 ; 1)$.

\subsection{Case 2}

For $|\delta Q|<\varepsilon$, at results obtained earlier in the paper keep valid because $\delta Q$ is in the nominator of the formula for calculating $d S$.

\subsection{Case 3}

Consider a general case where the infinitesimal quantity of heat $|\delta Q|$ can be not only less than or equal to the temperature difference $\varepsilon$ but also greater than that. Proceed from that if in some neighborhood of point $(0,0)$ and plane $0|\delta Q| \varepsilon$ the inequality is fulfilled

$|\delta Q| \leq k \varepsilon$

then a positive number $\delta_{1}$ can be found so that for all $|\delta Q| \leq \delta_{1}$ Eq. (4) is correct. It is clear that in this case all points of region $\Omega_{2}$ at $0<|\delta Q| \leq \delta_{1}$ lie above the straight line $|\delta Q|=k \varepsilon$ on the plane $0|\delta Q| \varepsilon$ (Figure 2).

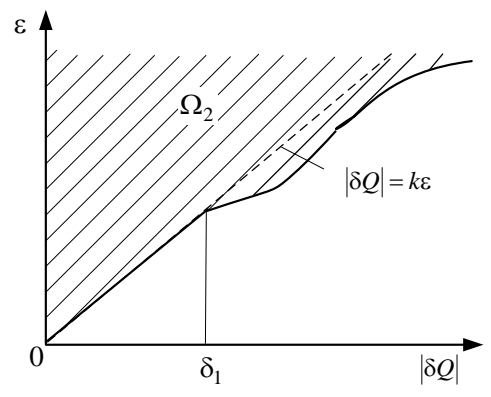

Figure 2. Region of fulfilling inequality (4) at $|\delta Q| \leq \delta_{1}$.

It is easy to see that the results obtained earlier in this paper become particular cases of condition (4) at $k=1$ and $k<1$, respectively.

If however the axis $0 T$ at the point $(0,0)$ touches the region $\Omega_{3}$ on the plane $0 \varepsilon T$ and in particular under the condition that $\Omega_{3}$ is bounded by the axis $0 T$ and the graph of the function described by (3), then with accounting for the condition (4) we obtain the following:

$\left.d S\right|_{T \rightarrow 0}=\left.\frac{\delta Q}{T}\right|_{T \rightarrow 0} \leq-\left.\frac{k \varepsilon}{\varepsilon \gamma(\varepsilon)}\right|_{\varepsilon \rightarrow 0} \rightarrow 0$.

In this case the condition $\varepsilon \gamma(\varepsilon) \rightarrow 0$ means that $T \rightarrow 0$ when the point $(\varepsilon, T) \rightarrow(0,0)$ while lying at the graph of the function (3). Then also considering $T(0)=\lim _{\varepsilon \rightarrow 0} \varepsilon \gamma(\varepsilon)=0$, we have

$T^{\prime}(0)=\lim _{\varepsilon \rightarrow 0+0} \frac{T(\varepsilon)-T(0)}{\varepsilon-0}=\lim _{\varepsilon \rightarrow 0+0} \frac{\varepsilon \gamma(\varepsilon)-0}{\varepsilon}=\lim _{\varepsilon \rightarrow 0} \gamma(\varepsilon)=+\infty$

that is the graph of the function $T(\varepsilon)$ touches the axis $0 T$ at the right side (Figure 3 ).

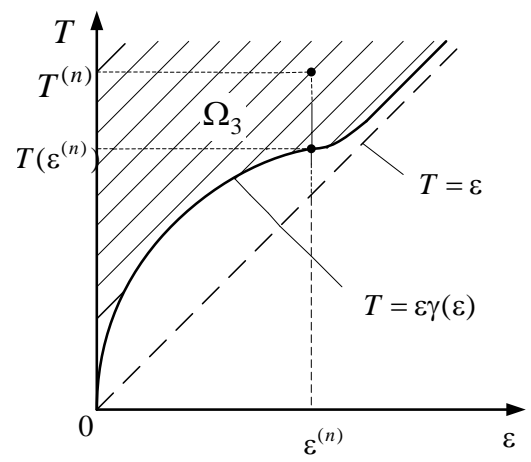

Figure 3. Region of fulfilling limit (7).

Because all limits are found at $|\delta Q|>0, \varepsilon>0$, then it is clear that the so-called "right-hand" tangent is considered, i.e. the tangent at $|\delta Q| \rightarrow 0+0$ in $0|\delta Q| \varepsilon$ or at $\varepsilon \rightarrow 0+0$ in $0 \varepsilon T$.

If however, we take some sequence of points $\left(\varepsilon^{(n)}, T^{(n)}\right) \rightarrow(0,0)$ in the region $\Omega_{3}$, then we have

$\lim _{n \rightarrow \infty} \frac{T^{(n)}-0}{\varepsilon^{(n)}-0} \geq \lim _{n \rightarrow \infty} \frac{T\left(\varepsilon^{(n)}\right)-0}{\varepsilon^{(n)}-0}=\lim _{\varepsilon \rightarrow 0} \frac{T(\varepsilon)-0}{\varepsilon-0}=T^{\prime}(0)=+\infty$

because the graph of function $T(\varepsilon)$ touches at the right-hand side the axis $0 T$ at the point $(0,0)$.

If however the point $(|\delta Q|, \varepsilon)$ approaches the origin $(0,0)$ while being in the region $\Omega_{4}$ touching the axis $0 \varepsilon$ at the point $(0,0)$, for example, if the region $\Omega_{4}$ is bounded by the axis $0 \varepsilon$ and by the graph of the function $\varepsilon=\phi(|\delta Q|)$ having at the point $|\delta Q|=0$ a vertical tangent, i.e. if $\phi(0)=0$ and

$\phi^{\prime}(|\delta Q|)=\lim _{|\delta Q| \rightarrow 0+0} \frac{\phi(|\delta Q|)}{|\delta Q|}=+\infty$,

then for any $T>\varepsilon$ so that $(\varepsilon, T)$ approaches the origin $(0,0)$ in the region $\Omega_{5}$ at $|\delta Q| \rightarrow 0$, we obtain in particular the following: 


$$
\begin{aligned}
& \left.d S\right|_{T \rightarrow 0}=\left.\frac{\delta Q}{T}\right|_{T \rightarrow 0}=\left.\frac{\delta Q}{\phi(|\delta Q|)} \cdot \frac{\phi(|\delta Q|)}{T}\right|_{T \rightarrow 0}< \\
& \left.\frac{\delta Q}{\phi(|\delta Q|)} \cdot \frac{\phi(|\delta Q|)}{\varepsilon}\right|_{\varepsilon \rightarrow 0,|\delta Q| \rightarrow 0} \leq\left.\frac{\delta Q}{\phi(|\delta Q|)}\right|_{\delta Q \rightarrow 0} \\
& =\left.\frac{1}{\phi(|\delta Q|) / \delta Q}\right|_{|\delta Q| \rightarrow 0} \rightarrow 0
\end{aligned}
$$

In Eq.(9) it is accounted for that the region $\Omega_{4}$ is specified by the inequality $\phi(|\delta Q|)<\varepsilon$ and the region $\Omega_{5}$ is specified by the inequality $T>k \varepsilon$ (Figure 4,5 ).

The reasoning given above are still valid for the region $\Omega_{6}$, for which $T>k \varepsilon$ at $\varepsilon<\delta_{2}$ (where $\delta_{2}>0$ is some positive number) (Figure 6).

It is easy to see that the region $\Omega_{6}$ of interdependence of $T$ and $\varepsilon$ is more wide than the region $\Omega_{5}$.

Obviously, $d S$ is a function differentiable with respect to the variables $T, \varepsilon$ and $|\delta Q|$ in the abovementioned regions.

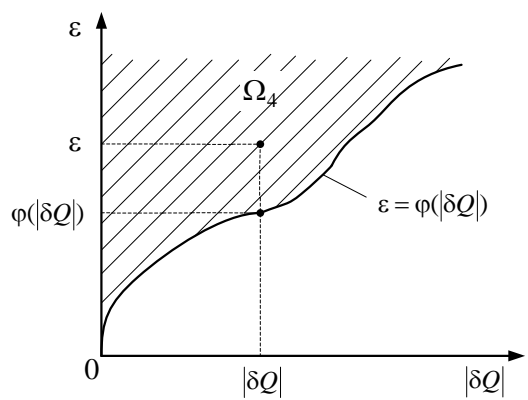

Figure 4. Region $\Omega_{4}$ of interdependence of $\varepsilon$ and $|\delta Q|$.

Then from Eqs. ( $\left.2^{\prime}\right),(5)$ and (9) it follows that at $T \rightarrow 0$ along the curve $T=T(\varepsilon)$ the entropy $S$ of the body being cooled approaches a constant value:

$\left.S\right|_{T \rightarrow 0} \rightarrow S_{0}=$ const.

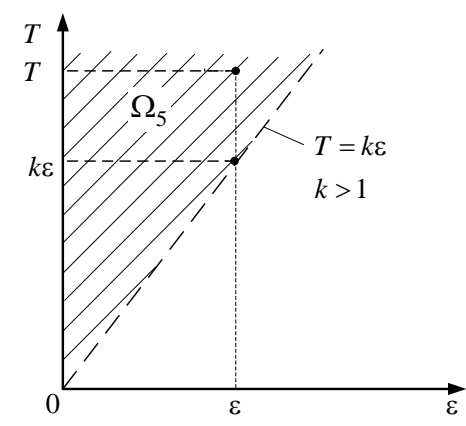

Figure 5. Region $\Omega_{5}$ of interdependence of $T$ and $\varepsilon$.

Thereby, the Nernst heat theorem has been proved analytically.

The constant in the right-hand side of (10) equals $S(0,0)$ given the entropy $S$ is finite at the point $(0,0)$. In particular, if $S(0,0)=0$ then the entropy $S \rightarrow 0$ at $(\varepsilon, T) \rightarrow(0,0)$ in $\Omega_{1}, \Omega_{3}$ at $\varepsilon$ and $|\delta Q|$ changing in the regions $\Omega_{2}, \Omega_{4}$, furthermore for $\Omega_{4}$, generally speaking, in a more broad class of regions including for example, the regions like $\Omega_{5}$ and $\Omega_{6}$. This conclusion does not contradict the well-known supposition by M. Planck to set the entropy $S$ to zero at the point $(0,0)$.

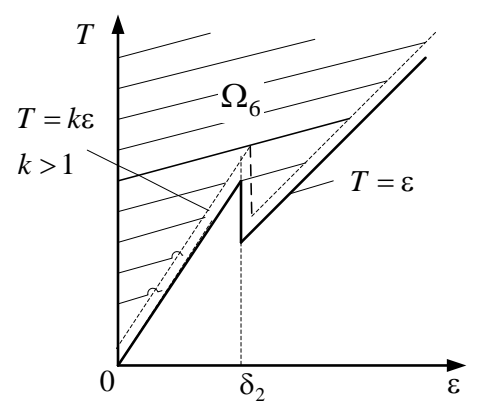

Figure 6. Region $\Omega_{6}$ of interdependence of $T$ and $\varepsilon$.

From Eq. (10) it follows that at $T \rightarrow 0$ bodies become unable to exchange heat and the absolute zero temperature is unachievable.

Therefore, in the above-stated it was proved that in the regions considered, the value of $d S$ is infinite small including $T \rightarrow 0$. Then as extreme transition we obtain the following:

$$
\lim _{T \rightarrow 0} \delta Q=\lim _{T \rightarrow 0}(T d S)=\lim _{T \rightarrow 0} T \cdot \lim _{T \rightarrow 0} d S=0 \cdot 0=0
$$

From the formula (11) is follows that when temperatures in the considered regions approach absolute zero, the infinitesimal quantity of heat is infinite small of a higher order than any of the multipliers $T$ and $d S$ in (11), i.e. it becomes arbitrarily small.

\subsection{Theoretical of Corollaries from Nernst Heat Theorem Proof}

Consider, finally, the behavior of the heat capacity $C(T)$ when approaching the absolute zero temperature, and for this purpose perform analysis of a known formula to determine it:

$$
C(T)=\frac{\delta Q}{d T}
$$

By the definition of the differential of the independent variable at the point $\mathrm{T}=0$ the relation is fulfilled $d T=T-0=T$ and instead of (12), the equality is obtained

$$
\left.C(T)\right|_{T \rightarrow 0}=\frac{\delta Q}{T}
$$

from which based on ( $\left.2^{\prime}\right),(5)$ and (9) it follows that at $T \rightarrow 0$ in the regions $\Omega_{2}$ and $\Omega_{4}$, the body heat capacity $C(T)$ also approaches zero:

$\left.C(T)\right|_{T \rightarrow 0} \rightarrow 0$

The result obtained above applies in particular to the isochoric $C_{V}$ and isobaric $C_{p}$ heat capacities, because it is not connected with the conditions of the heat exchange process. From this it follows first of all that at $T \rightarrow 0$ the heat capacities $C_{V}$ and $C_{p}$ become indistinguishable from each other. Moreover, in the known formulas for their 
determining in respect to the thermo deformation system $C_{V}=\left(\frac{\partial U}{\partial T}\right)_{\mathrm{v}}$ and $C_{p}=\left(\frac{\partial H}{\partial T}\right)_{p}$ at $T \rightarrow 0$ the conjugation conditions $V=$ const and $p=$ const can be omitted and the following can be written:

$$
C_{V}=\frac{d U}{d T} \quad \& \quad C_{p}=\frac{d H}{d T}
$$

Then based on the formula (15), we obtain correspondingly

$\left.\left.\frac{d U}{d T}\right|_{T \rightarrow 0} \rightarrow 0 \quad \& \quad \frac{d H}{d T}\right|_{T \rightarrow 0} \rightarrow 0$

Therefore, at the temperature approaching absolute zero, the internal energy of the body $U$ and its enthalpy $H$ approach the constant values undistinguishable from each other. The free internal energy $F=U-T \cdot S$ and free enthalpy $\Phi=H-T \cdot S$ also approach these values.

\section{Closing Remarks}

As a part of phenomenological view the proof of Nernst heat theorem and its corollaries is obtained.

\section{References}

[1] A.H. Wilson, Thermodynamics and Statistical Mechanics. Cambridge University Press. London, 1957.

[2] R. Kubo, Statistical Mechanics. North-Holland Publishing Company. Amsterdam, 1965.

[3] M. Tribus, Thermostatics and Thermodynamics. D. Van Nostrand Company, Princeton, New Jersey, 1961.

[4] R. Kubo, Thermodynamics. North-Holland Publishing Company. Amsterdam, 1968. 